\title{
Acumulación de masa ósea medida por DEXA periférica en niños mexicanos sanos de entre 6 a 12 años de edad
}

\author{
Ericka Montijo-Barrios, MC, (I) Jaime Ramírez-Mayans, MC,(I) Margarita García-Campos, LN, ${ }^{(1)}$ Rodrigo Díaz, PhD, (2) \\ Guillermo Sólomon-Santibañez, MC, (I) Pedro Gutiérrez-Castrellón, Dr en C.(I)
}

\begin{abstract}
Montijo-Barrios E, Ramírez-Mayans J, García-Campos M, Díaz R, Sólomon-Santibañez G, Gutiérrez-Castrellón P. Acumulación de masa ósea medida por DEXA periférica en niños mexicanos sanos de entre 6 a 12 años de edad.
\end{abstract} Salud Publica Mex 2009;5 I:227-230.

\section{Resumen}

Objetivo. Conocer la densidad mineral ósea (DMO), evaluada a través de DEXA periférica, ajustada por sexo, en una muestra de escolares mexicanos sanos de entre 6 a 12 años de edad. Material y métodos. Estudio transversal efectuado en I 965 escolares (982 masculinos), seleccionados de forma aleatoria en la Ciudad de México en 2006 y 2007. Se aplicó estadística descriptiva para variables nominales o categóricas y numéricas. Resultados. La curva de cambio de la DMO en niñas muestra dos etapas: un crecimiento lineal (entre los 6 a 9 años de edad) y uno exponencial (a partir de los 10 años), mientras que en los niños las tasas de crecimiento son menos pronunciadas. Conclusiones. Las intervenciones para mejorar la DMO y el pico máximo de masa ósea deben realizarse entre los 6 a los 12 años de edad.

Palabras clave: masa ósea; densitometría; niños; DEXA; México
Montijo-Barrios E, Ramírez-Mayans J, García-Campos M, Díaz R, Sólomon-Santibañez G, Gutiérrez-Castrellón P. Bone mass accretion in healthy Mexican

6 to 12 year-olds measured by DEXA. Salud Publica Mex 2009;5 I:227-230.

\section{Abstract}

Objective. To describe bone mass density in a group of healthy 6 to 12 year-old Mexican children by a peripheral DEXA, adjusted for gender. Material and Methods. A cross-sectional study between 2006 and 2007 conducted in I 965 children (982 male), without any medical condition, randomized from elementary schools in Mexico City. Results. Changes in bone mineral density in girls show two phases: a lineal accretion (6-9 years) and an exponential curve ( 10 years and older); in boys these growth rates are less pronounced. Conclusions. It is considered that optimal interventions to improve bone mass density and peak bone mass should be performed between 6 to 12 years of age.

Key words: bone mass, densitometry, Mexican children, DEXA

(I) Instituto Nacional de Pediatría. México, DF, México.

(2) Instituto Nacional de Salud Pública. Cuernavaca, Morelos, México.

Fecha de recibido: 10 de octubre de 2008 • Fecha de aprobado: 16 de febrero de 2009

Solicitud de sobretiros: Dr. Pedro Gutiérrez-Castrellón. Primer Piso,Torre de Investigación. Dirección de Investigación. Instituto Nacional de Pediatría. Insurgentes Sur 3700-C, Cuicuilco. 04530 Coyoacán, México, DF. México.

Correo electrónico: inpcochrane@gmail.com 
E depósito de masa ósea durante la niñez y la adolescencia depende de diversos factores, entre ellos los genéticos, sexo, estilo de vida y aspectos conductuales y ecológicos, los cuales determinan la tasa definida de depósito de tejido óseo y la maduración de las áreas de remodelación y el esqueleto en su totalidad. ${ }^{1-6}$ Este estado de desarrollo de la masa ósea se conoce como remodelación ósea y se trata de un proceso que ocurre a un ritmo acelerado durante la preadolescencia y por lo general se completa durante la fase 4 del estadio de desarrollo de Tanner, que inicia habitualmente a los 10 años de edad en las niñas y a los 14 años en los niños. ${ }^{6-14}$ Después de este periodo, la remodelación ósea continúa lentamente hasta que se alcanza el pico de masa ósea. 9-20 En realidad, evidencia creciente muestra que diversas intervenciones dirigidas a optimizar el pico de masa ósea antes de la fase 4 de Tanner representan una ventana de oportunidad para prevenir la osteoporosis futura., 7,21 La absorciometría de energía dual por rayos X (DEXA) es el estándar de referencia para definir la tasa de mineralización en el esqueleto total y las diferentes regiones, a través de la evaluación denominada densidad mineral ósea (DMO). ${ }^{21-28}$ Con base en lo anterior, el objetivo de este estudio fue conocer la DMO en una muestra de escolares mexicanos sanos de entre 6 a 12 años de edad, evaluada a través de DEXA, ajustada por sexo, con la finalidad de analizar el comportamiento de la curva de DMO y sus cambios a lo largo del tiempo.

\section{Material y métodos}

Éste es un estudio transversal, aprobado por los Comités de Investigación y Ética del Instituto Nacional de Pediatría y realizado en escuelas primarias de la Ciudad de México; se empleó un muestreo aleatorio, polietápico y en conglomerados. Para tal efecto se identificaron las primarias de las distintas delegaciones del Distrito Federal (DF) y municipios del Estado de México. Todas ellas se zonificaron y dividieron en cuatro secciones. En cada sección se enumeraron las primarias en orden consecutivo y se seleccionó cada primaria en forma aleatoria simple (tabla de números aleatorios). De acuerdo con el número de escolares en cada primaria, se efectuó un estimado proporcional. Cada niño se enumeró en forma consecutiva y mediante selección aleatoria se designó a los niños con posibilidad de incluirse. Participó un total de nueve escuelas.

Mediante interrogatorio médico y exploración física de la población elegible, se identificaron aquellos escolares que no presentaran afección evidente y que aceptaron previamente participar junto con sus padres en el estudio. Se excluyó a un total de cinco niños (dos hijos de extranjeros y tres niños con tratamiento farma- cológico que interfería con el metabolismo del calcio). En todos los casos se contó con consentimiento informado, el cual fue firmado por ambos padres. De la misma forma, se obtuvo el asentimiento del menor cuando así fue requerido. Ambas cartas fueron revisadas y aprobadas por los Comités de Ética e Investigación del Instituto Nacional de Pediatría.

Se efectuó exploración física completa para descartar enfermedad evidente mediante toma de signos vitales y revisión por segmentos corporales. Se realizó una medición del peso, talla, perímetro braquial y estimación de la grasa corporal total mediante impedancia bioeléctrica (TANITA Analyzer mod. BF-350). Con dichas mediciones se obtuvieron los indicadores antropométricos: índice de masa corporal, peso para la talla, talla para la edad y porcentaje de grasa.

La medición de la DMO, expresada en $\mathrm{g} / \mathrm{cm}^{2}$, se efectuó en el antebrazo no dominante mediante un instrumento DEXA Lunar PIXI periférico; se consideró una calibración in vivo de $\mathrm{VC}<1.8 \%$ e in vitro de $\mathrm{VC}<1.5 \%$. El Lunar PIXI cuenta con una técnica de absorciometría de doble energía, con detector de área estacionaria y fuente de rayo en cono, lo que permite eliminar el barrido mecanizado. Los resultados se procesaron mediante equipo Celeron a $2 \mathrm{GHZ}$ de $128 \mathrm{MB}$ en RAM. Todas las mediciones se llevaron a cabo previa estandarización de los investigadores. Desde el punto de vista estadístico, se efectuó una descripción de las variables nominales o categóricas mediante porcentajes y de las variables numéricas a través de promedio \pm desviación estándar o a través de mediana (mínimos-máximos); para el cálculo se utilizó el programa estadístico SPSS versión 16.0 para MAC.

\section{Resultados}

Se incluyó en el estudio a un total de 1965 niños mestizos mexicanos de entre 6 a 12 años de edad, de los cuales 982 (42\%) fueron del sexo masculino. Las mediciones absolutas de la DMO permiten identificar valores diferenciales acordes con el sexo y la edad de los niños incluidos en el estudio (cuadro I). Al analizar la curva de cambio de la DMO de acuerdo con el sexo, se observa que la gráfica de las niñas muestra dos etapas importantes. Primero se reconoce un crecimiento lineal que se inicia antes de los seis años y termina a los nueve años; en esta etapa la tasa relativa de crecimiento fluctúa entre $0.39 \%$ (siete años) y $0.81 \%$ (nueve años). A este crecimiento lineal le sigue una etapa de crecimiento exponencial; a los 10 años la tasa cambia súbitamente a $2.06 \%$ (se incrementa casi tres veces) hasta los 12 años, cuando se observa una tasa de $7.50 \%$, mientras que en los niños la tasa de crecimiento a partir de los nueve años es menos pronunciada (figura 
1). Al analizar las tasas relativas de cambio de la DMO se identifica de manera similar un ajuste por género y edad (cuadro II). Al efectuar el análisis de la curva de mediciones relativas de la $\mathrm{DMO}$, se puede advertir cómo en ambos sexos se evidencia un crecimiento exponencial de la DMO. En las niñas, esta etapa se presenta un año antes que en los niños (10 contra 11 años) con tasas sin diferencias significativas $(2.06 \%$ para las niñas y $2.46 \%$ para los niños). Sin embargo, a los 12 años la tasa de las niñas es mayor que la de los niños (7.5 contra $4.5 \%$ ) (figura 2). Al comparar los resultados por sexo, se observa cómo, a pesar de que a los seis años la DMO en la niñas es menor (niñas, $0.293 \mathrm{~g} / \mathrm{cm}^{2}$; niños, $0.313 \mathrm{~g} / \mathrm{cm}^{2}$ ), debido al retraso del crecimiento exponencial presente en

Cuadro I

Densidad mineral ósea* del antebrazo, por seXo. Ciudad de México, 2006-2007

$\begin{array}{cccc}\text { Edad } & \text { Femenino } & \text { Masculino } & \text { Diferencia } \\ \text { (años) } & D M O\left(\mathrm{~g} / \mathrm{cm}^{2}\right) & \mathrm{DMO}\left(\mathrm{g} / \mathrm{cm}^{2}\right) & \text { entre sexos }\end{array}$

\begin{tabular}{rrrr}
6 & 0.293 & 0.313 & -0.019 \\
\hline 7 & 0.295 & 0.311 & -0.017 \\
\hline 8 & 0.296 & 0.310 & -0.014 \\
\hline 9 & 0.298 & 0.310 & -0.012 \\
\hline 10 & 0.305 & 0.313 & -0.009 \\
\hline 11 & 0.318 & 0.321 & -0.003 \\
\hline 12 & 0.342 & 0.335 & 0.006
\end{tabular}

$* \mathrm{~g} / \mathrm{cm}^{2}$

DMO: densidad mineral ósea

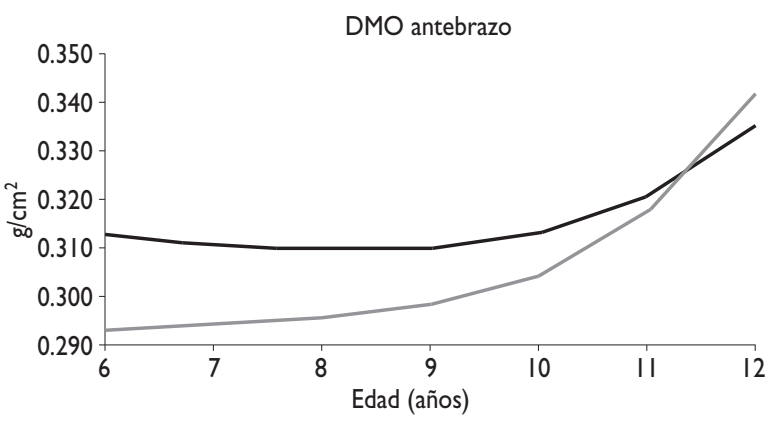

- Masculino $\quad$ Femenino

Figura I. Curva de densidad mineral ósea AJustada por EDAD Y SEXO. CIUDAD DE MÉXICO, 2006-2007
Cuadro II

TASA Relativa de CRECIMIENTO de LA DENSIDAD mineral ósea. Ciudad de MéXico, 2006-2007

\begin{tabular}{ccc}
$\begin{array}{c}\text { Edad } \\
\text { (años) }\end{array}$ & $\begin{array}{c}\text { Femenino } \\
(\%)\end{array}$ & $\begin{array}{c}\text { Masculino } \\
(\%)\end{array}$ \\
7 & 0.39 & -0.57 \\
\hline 8 & 0.51 & -0.31 \\
\hline 9 & 0.81 & 0.05 \\
\hline 10 & 2.06 & 0.94 \\
\hline 11 & 4.36 & 2.46 \\
\hline 12 & 7.50 & 4.50 \\
\hline
\end{tabular}

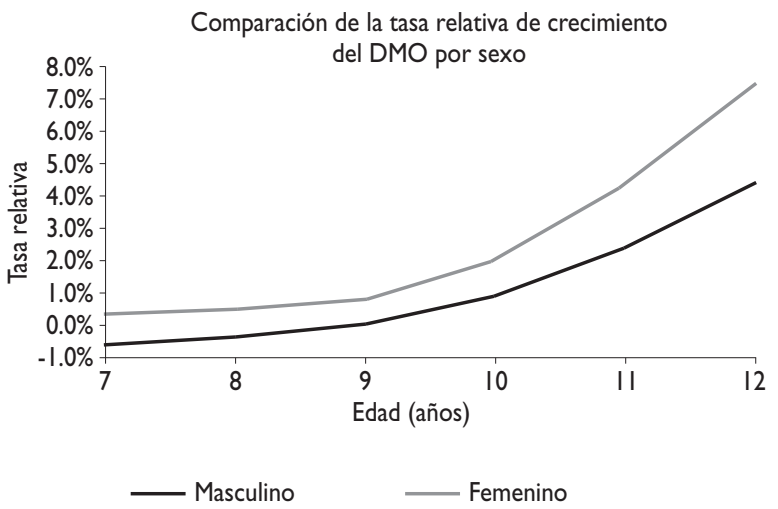

Figura 2. TASA Relativa de densidad Mineral ÓSEA, POR SEXo. CIUdAd DE MéXICO, 2006-2007

los niños, a los 12 años la $\mathrm{DMO}$ es discretamente mayor en las niñas (niñas, $0.342 \mathrm{~g} / \mathrm{cm}^{2}$; niños, $0.335 \mathrm{~g} / \mathrm{cm}^{2}$ ). El crecimiento exponencial se inicia a los 12 años en las niñas $(0.25 \%)$ y a los 13 años en los niños (0.39\%). En el intervalo de los 13 a 14 años, la tasa prácticamente se ha duplicado (de 0.51 a $0.91 \%$ en las niñas y de 0.39 a $0.87 \%$ en los niños). En los niños, el intervalo de variación de las tasas de DMO oscila entre -0.57 y $4.50 \%$.

\section{Discusión}

Los datos obtenidos muestran que la acumulación de la DMO inicia de forma lenta después de los ocho años de edad en las niñas y con una clara aceleración a los 10 años. En los niños, la acumulación de la DMO en ambas fases inicia un año después. El depósito de tejido óseo y su mineralización con cristales amorfos de fosfato de calcio es necesario para conseguir la maduración nor- 
mal de la hidroxiapatita y la consolidación de la masa ósea. Se requieren al menos cuatro meses para que los cristales de fosfato de calcio se depositen, conformen la matriz del hueso de todo el esqueleto y se conviertan de forma subsecuente en la hidroxiapatita madura. De los seis a los 10 años de edad la DMO es significativamente mayor en niños que en niñas (figura 1), si bien el rápido incremento de la DMO de las niñas a partir de los 10 años da lugar a que ésta sea similar a la de los niños para los 11 años y que se la supere a los 12 años.

En el cuadro II se observa que los niños mostraron a los 12 años una tasa anual de $4.5 \%$, mientras que en las niñas dicha tasa anual es de $7.5 \%$, lo cual explica el comportamiento de la DMO en esta etapa. Lo anterior se correlaciona con el hecho de que los niños durante este periodo tienen una maduración sexual y esteroidea más lenta que las niñas; los incrementos de la DMO pueden medirse mediante DEXA en zonas específicas cuando los miligramos de hidroxiapatita $/ \mathrm{cm}^{2}$ se han distribuido en todo el esqueleto. Para optimizar las estrategias en el seguimiento de la DMO en escolares (incluidas las evaluaciones nutricionales y de actividad física) es necesario determinar los cambios en la DMO cada año después de los ocho años de edad, cuando el cambio de comportamiento de la curva refleja la ganancia de la hidroxiapatita en el hueso. Por lo tanto, para mejorar el depósito de masa ósea es preciso realizar intervenciones oportunas mediante orientación alimentaria y promoción de la actividad física entre los 8 y 14 años de edad para ambos sexos.

\section{Referencias}

I. Leonard MB, Zemel BS. Currents concepts in pediatric bone disease. Pediatr Clin North Am 2002;49(I): I43-I73.

2.PollitzerWS,Anderson JJB. Ethnic and genetic differences in bone mass: a review with a hereditary vs environmental perspective. Am J Clin Nutr 1989; 50:1244-1259.

3. Slemenda CW, Miller JZ, Hui SL, Reister TK, Johnston CCJr. Role of physical activity in the development of skeletal mass in children.J Bone Miner Res 1991;6:1227-1233.

4. Soroko S, Holbrook TL, Edelstein S, Barrett-Connor E. Lifetime milk consumption and bone mineral density in older women. Am J Public Health 1994;84:1319-1322.

5. Bonjour JP, Carrie AL, Ferrari S, Clavien H, Slosman D, Theintz G, et al. Calcium enriched foods and bone mass growth in prepubertal girls: a randomized, double-blind, placebo controlled trial.J Clin Invest 1997;99: I 287-I 294.

6. Chan GM, Hoffman K, McMurry M. Effects of dairy products on bone and body composition in pubertal girls. J Pediatr 1995;126: I- 12.

7. Johnston CC Jr, Miller JZ, Slemenda CW, Reister TK, Hui S, Christian JC, et al. Calcium supplementation and increases in bone mineral density in children. N Engl J Med 1992;387:82-87.

8. Lee WT, Leung SS, Wang SH, XuYC, Zeng WP, Lau J, et al. Double - blind, controlled calcium supplementation and bone mineral accretion in children accustomed to a low calcium diet. Am J Clin Nutr 1994;60:744-750.
9. Lee WT, Leung SS, Leung DM,Wang SH, XuYC, Zeng WP, et al. Bone mineral acquisition in low calcium intake children following the withdrawal of calcium supplement. Acta Paediatr 1997;86:570-576. 10. Bailey DA, McKay HA, Mirwald RL, Crocker PR, Faulkner RA.A sixyear longitudinal study of the relationship of physical activity to bone mineral accrual in growing children:The University of Saskachewan bone mineral accrual study.J Bone Miner Res 1999; 14: 1672-1679.

I I. Goulding A, Jones IE, Taylor RW, Manning PJ,Williams SM. More broken bones: a 4-year double-cohort study of growing girls with and without distal forearm fractures. J Bone Miner Res 2000;15: 201 I-2018.

12. Manolagas SC. Role of cytokines in bone resorption. Bone 1995; I7(Suppl): 63-67.

13. Brown J], Zacharin MR. Proposals for prevention and management of steroid induced osteoporosis in children and adolescents.J Pediatr Child Health 2005;4I: 553-557.

14. Hansen M, Halberg P, Kollerup G, Pedersen-Zbinden B, Hørslev-

Petersen K, Hyldstrup L, et al. Bone metabolism in patients with systemic lupus erythematosus. Scand J Rheumatol 1998;27:197-206.

15. Kipen Y, Strauss BJ, Morand EF. Body composition in systemic lupus erythematosus. Br J Rheumatol 1998;37:514-519.

16. Kipen Y, Briganti EM, Strauss BJ, Littlejohn GO, Morand EF.Three year follow-up of body composition changes in pre-menopausal women with systemic lupus erythematosus. Rheumatology 1999;38:59-65.

17. Dhillon VB, Davies MC, Hall ML, Round JM, Ell PJ, Jacobs HS, et al. Assessment of the effect of corticosteroids on bone mineral density in systemic lupus erythematosus: a preliminary study with Dual energy X-ray absorptiometry. Ann Rheum Dis 1990; 49: 624-626.

18. Kalla AA, Fataar AB, Jessop SJ, Bewerunge L. Loss of trabecular bone mineral density in systemic lupus erythematosus. Arthritis Rheum 1993;36:1726-1734.

19.Araüjo NC, SzejnfeldVL, Sato El, Munhoz MC, Atra E. The effect of oral corticosteroid (CE) on bone mineral density (BMD) in systemic lupus erythematosus (SLE). Rev Bras Rheumatol 1994;S6I (Abstract).

20. Formiga F, Moga I, Nolla JM, Pac M, Mitjavila F, Roig-Escofet D. Loss of bone mineral density in premenopausal women with systemic lupus erythematosus. Ann Rheum Dis 1995;54:274-276.

21. Houssiau FA, Lefebvre C, Depresseux G, Lambert M, Devogelaer JP, Nagant de Deuxchaisnes C. Trabecular and cortical bone loss in systemic lupus erythematosus. Br J Rheumatol 1996; 35:244-247.

22. Sels F, Dequeker J,Verwilghen J, Mbuyi-Muamba JM. SLE and osteoporosis: dependence and / or independence on glucocorticoids. Lupus 1996;5:89-92.

23. Sinigaglia L,Varenna M, Binelli L, Zucchi F, Ghiringhella D, Galazzi M, et al. Determinants of bone mass in systemic lupus erythematosus: $A$ cross sectional study on premenopausal women.J Rheumatol 1999;26: 280- 1284.

24. Lakshminarayanan S, Walsh S, Mohanraj M, Rothfield N. Factors associated with low bone mineral density in female patients with systemic lupus erythematosus.J Rheumatol 2001;28:102-108.

25. Yee CS, Crabtree N, Skan J,Amft N, Bowman S, Situnayake D, et al. Prevalence and predictors of fragility fractures in systemic lupus erythematosus. Ann Rheum Dis 2005;64: I I I- I I3.

26. Trapani S, Civinini R, Ermini M, Paci E, Falcini F. Osteoporosis in juvenile systemic lupus erythematosus: a longitudinal study on the effect of steroids on bone mineral density. Rheumatol Int 1998; I8:45-49.

27. Chinn DJ, Fordham JN, Kibirige MS, Crabtree NJ,Venables J, Bates J, et al. Bone density at the os calcis: reference values, reproducibility, and effects of fracture history and physical activity.Arch Dis Child 2005;90:30-35. 28.Van der Sluis IM, de Ridder MA, Boot AM, Krenning EP, de Muinck Keizer-Schrama SM. Reference data for bone density and body composition measures with dual energy $\mathrm{x}$ ray absorptiometry in white children and young adults. Arch Dis Child 2002;87:34I-347. 